\title{
Lattice Boltzmann model for thermal transpiration
}

\author{
G. H. Tang* \\ Computational Science and Engineering Department, STFC Daresbury Laboratory, Warrington, WA4 4AD, United Kingdom \\ Y. H. Zhang ${ }^{\dagger}$ \\ Department of Mechanical Engineering, University of Strathclyde, Glasgow, G1 1XJ, United Kingdom \\ X. J. Gu, R. W. Barber, and D. R. Emerson \\ Computational Science and Engineering Department, STFC Daresbury Laboratory, Warrington, WA4 4AD, United Kingdom
}

(Received 19 September 2008; revised manuscript received 24 December 2008; published 12 February 2009)

\begin{abstract}
The conventional Navier-Stokes-Fourier equations with no-slip boundary conditions are unable to capture the phenomenon of gas thermal transpiration. While kinetic approaches such as the direct simulation Monte Carlo method and direct solution of the Boltzmann equation can predict thermal transpiration, these methods are often beyond the reach of current computer technology, especially for complex three-dimensional flows. We present a computationally efficient nonequilibrium thermal lattice Boltzmann model for simulating temperature-gradient-induced flows. The good agreement between our model and kinetic approaches demonstrates the capabilities of the proposed lattice Boltzmann method.
\end{abstract}

DOI: 10.1103/PhysRevE.79.027701

PACS number(s): 05.10.-a, 47.45.-n, 47.61.-k

\section{INTRODUCTION}

In 1879, Reynolds discovered that a gas will move along a solid surface due to inequalities of temperature and called the phenomenon thermal transpiration [1]. In the same year, Maxwell [2] independently developed a theory to explain this effect. The phenomenon of thermal transpiration (or thermal creep [3]) has recently been used to develop a MEMS-based multistage Knudsen compressor [4,5] where the gas moves from a cold chamber to a hot chamber and builds up a pressure difference across the compressor element. Thermal creep occurs in the opposite direction to the tangential heat flux; i.e., the flow is in the direction of increasing temperature.

The physical explanation of thermal creep has been presented by Sone [6]. It is assumed that equal numbers of molecules arrive at the wall from the hot and cold regions. Molecules arriving from the hot region will have, on average, a higher velocity than those arriving from the cold region. Since the molecules are reflected diffusively at the wall, the resultant force on the wall due to the molecular collisions acts towards the cold region. An equal and opposite force is felt by the gas molecules, giving rise to a flow towards the hot region. Once the fluid starts to creep along the wall, the moving fluid layer interacts with the stagnant fluid layers adjacent to it, inducing a boundary layer.

Unfortunately, the flow within a Knudsen compressor is very complex, involving internal recirculation, vortices at the capillary exit, and reverse thermal creep flow, leading to lower than expected pressure ratios across the compressor. Although kinetic approaches such as the direct simulation Monte Carlo (DSMC) method and direct solution of the Boltzmann equation can predict thermal transpiration, these

\footnotetext{
*guihua.tang@stfc.ac.uk

†yonghao.zhang@strath.ac.uk
}

methods are only able to simulate a section of the device and cannot be used to optimize the geometry of the entire compressor [5]. Moreover, kinetic methods are still beyond the reach of current computer technology for large-scale threedimensional simulations. In contrast, continuum-based methods such as the Navier-Stokes-Fourier (NSF) equations are computationally efficient, but are unable to accurately capture thermal transpiration phenomena beyond the slip-flow regime. Here, we report a nonequilibrium thermal lattice Boltzmann (LB) model which can satisfy the twin demands of computational efficiency and numerical accuracy for simulating rarefied gas flows in complex, industrially relevant geometries. General information on the LB method can be found in the literature-e.g., $[7,8]$.

\section{NONEQUILIBRIUM THERMAL LATTICE BOLTZMANN MODEL}

Conventional LB models are unable to capture nonlinear constitutive behavior close to a solid surface since they only retain velocity terms up to second order in the Hermite expansion of the equilibrium distribution function, as reported by Shan et al. [9]. However, this is not sufficient even for isothermal flows and it is usually necessary to retain terms up to fourth order in the Hermite expansion. Moreover, to describe nonequilibrium physics beyond the level of the NSF equations, it is necessary to consider terms up to fifth order in the Hermite expansion. Inevitably, this leads to a large number of discrete velocities, which increases the computational cost and may give rise to numerical stability problems.

To overcome these difficulties, we make use of the different relaxation rates for momentum and energy, and propose a different energy density distribution function $g$ to describe the evolution of the temperature field, as suggested by $\mathrm{He} e t$ $a l$. [10] for no-velocity-slip and no-temperature-jump hydrodynamics. Using this approach, it is only necessary to retain up to the third-order moments of the energy density distribu- 
tion function in order to describe heat fluxes beyond the NSF level. As a consequence, our previously reported nonequilibrium isothermal lattice Boltzmann model [11] should be sufficiently accurate to describe the evolution of the velocity field. In the present approach, as well as solving the evolution equation for the particle number density, we solve an additional equation for the energy density. The model relates the energy density distribution function to the number density distribution function via a flexible Prandtl number.

The evolution equation for the velocity field has previously been described by Zhang et al. [12]:

$$
\begin{aligned}
f_{k}(\mathbf{x} & \left.+\mathbf{e}_{k} \delta t, t+\delta t\right)-f_{k}(\mathbf{x}, t) \\
& =-\frac{1}{\tau}\left[f_{k}(\mathbf{x}, t)-f_{k}^{e q}(\mathbf{x}, t)\right]+\delta t \frac{\left(e_{k i}-u_{i}\right) F_{i}}{c_{s}^{2} \rho} f_{k}^{e q}(\mathbf{x}, t),
\end{aligned}
$$

where $f_{k}$ is the distribution function for the number density at position $\mathbf{x}$ and time $t, f_{k}^{e q}$ is the corresponding distribution function at equilibrium, $e_{k i}$ is the lattice velocity, $u_{i}$ is the macroscopic velocity, $c_{s}$ is the sound speed of the lattice fluid, $\rho$ is the density, $\tau$ is the nondimensional relaxation time for the number density distribution function, and $\delta t$ is the time step. The kinematic viscosity is calculated from $\nu=(\tau$ $-0.5) c_{s}^{2} \delta t$. Following He et al. [10], the energy density evolution equation is given by

$$
g_{k}\left(\mathbf{x}+\mathbf{e}_{k} \delta t, t+\delta t\right)-g_{k}(\mathbf{x}, t)=-\frac{1}{\tau_{t}}\left[g_{k}(\mathbf{x}, t)-g_{k}^{e q}(\mathbf{x}, t)\right],
$$

where $g_{k}$ is the distribution function for the energy density at position $\mathbf{x}$ and time $t, g_{k}^{e q}$ is the corresponding distribution function at equilibrium, and $\tau_{t}$ is the nondimensional relaxation time for the energy density distribution function. The thermal diffusivity is given by $\alpha=\left(\tau_{t}-0.5\right) c_{s}^{2} \delta t$, and the Prandtl number is determined from $\operatorname{Pr}=(\tau-0.5) /\left(\tau_{t}-0.5\right)$ $[12,13]$. Viscous heating effects have been neglected in the present model since they are usually insignificant in lowspeed gas flows in micro- and nanodevices.

Following the spirit of rational number approximation [14], we have developed an isothermal lattice Boltzmann model beyond the level of the Navier-Stokes equations [11]. For a two-dimensional, 13-velocity lattice model (D2Q13) [11], the lattice velocities are given by

$$
\begin{gathered}
e_{0}=0, \\
e_{k}=\left[\cos \left(\frac{(k-1) \pi}{2}\right), \sin \left(\frac{(k-1) \pi}{2}\right)\right] c, \quad k=1-4, \\
e_{k}=\left[\cos \left(\frac{(k-5) \pi}{2}+\frac{\pi}{4}\right), \sin \left(\frac{(k-5) \pi}{2}+\frac{\pi}{4}\right)\right] \sqrt{2} c, \\
k=5-8,
\end{gathered}
$$

$$
e_{k}=\left[\cos \left(\frac{(k-1) \pi}{2}\right), \sin \left(\frac{(k-1) \pi}{2}\right)\right] 2 c, \quad k=9-12
$$

where $c=\sqrt{2 R T}$ and $R$ is the gas constant. The equilibrium distribution function can be expressed as

$$
\begin{gathered}
f_{k}^{e q}=\rho \omega_{k}\left[1+\frac{e_{k i} u_{i}}{c_{s}^{2}}+\frac{\left(e_{k i} u_{i}\right)^{2}}{2 c_{s}^{4}}-\frac{u_{i} u_{i}}{2 c_{s}^{2}}+\frac{\left(e_{k i} u_{i}\right)^{3}}{2 c_{s}^{6}}\right. \\
\left.-\frac{3\left(e_{k i} u_{i}\right)\left(u_{i} u_{i}\right)}{2 c_{s}^{4}}\right], \\
\omega_{0}=\frac{3}{8} ; \quad \omega_{k}=\frac{1}{12}, \quad k=1-4 ; \quad \omega_{k}=\frac{1}{16}, \quad k=5-8 ; \\
\omega_{k}=\frac{1}{96}, \quad k=9-12 .
\end{gathered}
$$

The sound speed of the lattice fluid is given by $c_{s}^{2}=c^{2} / 2$. The same D2Q13 lattice model is used to solve the evolution equation for the energy density distribution function. At equilibrium, the energy density is given by $g_{k}^{e q}=\epsilon f_{k}^{e q}$, where $\epsilon=D R T / 2$ and $D$ is the number of physical dimensions [12].

As discussed in our previous work [15], gas-surface interactions have a significant impact on the mean free path of the gas in the near wall region and these interactions need to be taken into account to capture the flow characteristics in the Knudsen layer. Following previous work which accounts for the effect of the wall [15-17] and considering how temperature relates to viscosity and density, the local relaxation time in the thermal LB model can be determined as follows:

$$
\tau=\frac{\lambda}{\lambda_{0}} \frac{\rho_{0}}{\rho}\left(\frac{T}{T_{0}}\right)^{\omega-0.5} \sqrt{\frac{\pi}{8}} \frac{c}{c_{s}} \mathrm{Kn}_{0} N_{L}+\frac{1}{2},
$$

where $N_{L}=L / \delta y$ is the number of lattices over the characteristic length $L, \delta y$ is the lattice spacing, $\rho_{0}$ is the density at the reference temperature $T_{0}$, and $\mathrm{Kn}_{0}$ is the Knudsen number based on the mean free path $\lambda_{0}$ evaluated from $\lambda_{0}$ $=\left(\mu_{0} / p_{0}\right) \sqrt{\pi R T_{0} / 2}$, where $p_{0}$ is the pressure and $\mu_{0}$ is the dynamic viscosity. The coefficient $\omega$ depends on the molecular interaction model, with $\omega=0.5$ for hard-sphere interactions and $\omega=1$ for Maxwellian interactions. The local mean free path ratio $\lambda / \lambda_{0}$ can be solved separately depending on the specific geometric conditions [15-17]. The thermal relaxation time is determined from $\tau_{t}=(\tau-0.5) / \operatorname{Pr}+0.5$.

In this work, a kinetic boundary condition with a diffuse scattering kernel is employed. The unknown reflected distribution function $f_{k}$ on the wall can be determined from the incident distribution function $f_{k^{\prime}}$ as follows [18]:

$$
\begin{aligned}
f_{k}(\mathbf{x}, t+\delta t)= & \frac{\sum_{\left(\mathbf{e}_{k^{\prime}}-\mathbf{u}_{w}\right) \cdot \mathbf{n}<0}\left|\left(\mathbf{e}_{k^{\prime}}-\mathbf{u}_{w}\right) \cdot \mathbf{n}\right| f_{k^{\prime}}(\mathbf{x}, t+\delta t)}{\sum_{\left(\mathbf{e}_{k}-\mathbf{u}_{w}\right) \cdot \mathbf{n}>0}\left|\left(\mathbf{e}_{k}-\mathbf{u}_{w}\right) \cdot \mathbf{n}\right| f_{k}^{e q}\left(\mathbf{x}, \boldsymbol{\rho}_{w}, \mathbf{u}_{w}\right)} \\
& \times f_{k}^{e q}\left(\mathbf{x}, \boldsymbol{\rho}_{w}, \mathbf{u}_{w}\right),
\end{aligned}
$$

where $\mathbf{u}_{w}$ and $\rho_{w}$ are the velocity and density at the wall, respectively, and $\mathbf{n}$ is the unit normal. The above Maxwellian 


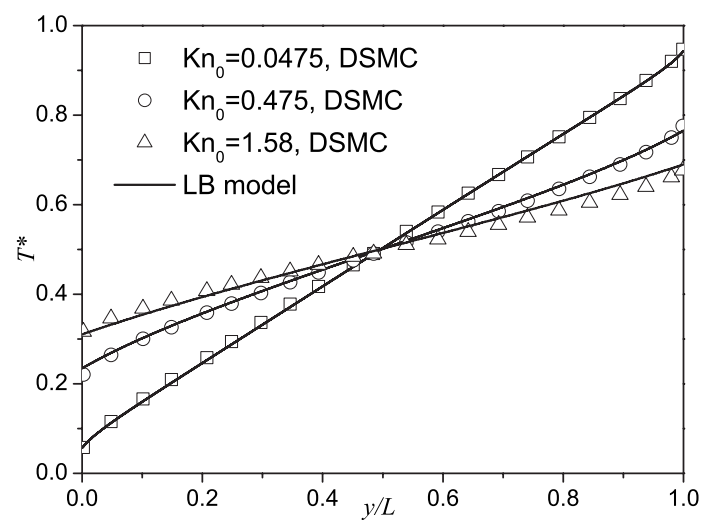

FIG. 1. Nondimensional temperature profiles for rarefied Fourier flow between two parallel plates. The symbols represent the DSMC data presented by Gallis et al. [19].

diffuse reflection at the wall assumes that the reflected particles are in thermal equilibrium with the wall. Since the discrete velocity amplitude in our D2Q13 model can be $2 c$, computational fluid particles that are two lattice spacings away from the wall may also hit the surface. This differs from standard LB models where only fluid particles at the nearest-neighboring grid points can interact with the wall. However, reflected fluid particles will only be at the nearest grid points to the wall and their velocity distribution is given by Eq. (6). The reflected energy density distribution can therefore be related to the reflected number density distribution as follows [12]:

$$
g_{k}=\frac{D R}{2} T_{w} f_{k}
$$

where $T_{w}$ is the temperature of the wall.

\section{RESULTS AND DISCUSSION}

First, we apply our LB model to rarefied Fourier flow between two parallel plates separated by a distance $L$. In the simulations, the temperatures of the two plates are maintained at $T_{1}$ and $T_{2}$, respectively, with $T_{2}>T_{1}$. The mean temperature $\left(T_{1}+T_{2}\right) / 2$ is used as a reference temperature $T_{0}$, and the Prandtl number is fixed at 0.68 for consistency with the DSMC data presented by Gallis et al. [19]. The nondimensional temperature is defined as $T^{*}=\left(T-T_{1}\right) /\left(T_{2}-T_{1}\right)$. Figure 1 clearly shows that the temperature jump at the wall increases with Knudsen number. At low Kn, the temperature profile is almost linear, but becomes increasingly nonlinear at higher Knudsen numbers. It can be seen that the LB model is in good agreement with the DSMC data in both the slip and transition regimes.

The second test case considers thermal creep flow between two parallel plates separated by a distance, $L$. Figure 2 shows the normalized velocity profiles across the channel at various Knudsen numbers and compares the results from our lattice Boltzmann model against the data obtained by Ohwada et al. [20] using a direct solution of the linearized Boltzmann equation. It can be seen that our LB model is in very good agreement with the solution from the linearized Boltzmann equation for Knudsen numbers up to $\mathrm{Kn}=0.677$,
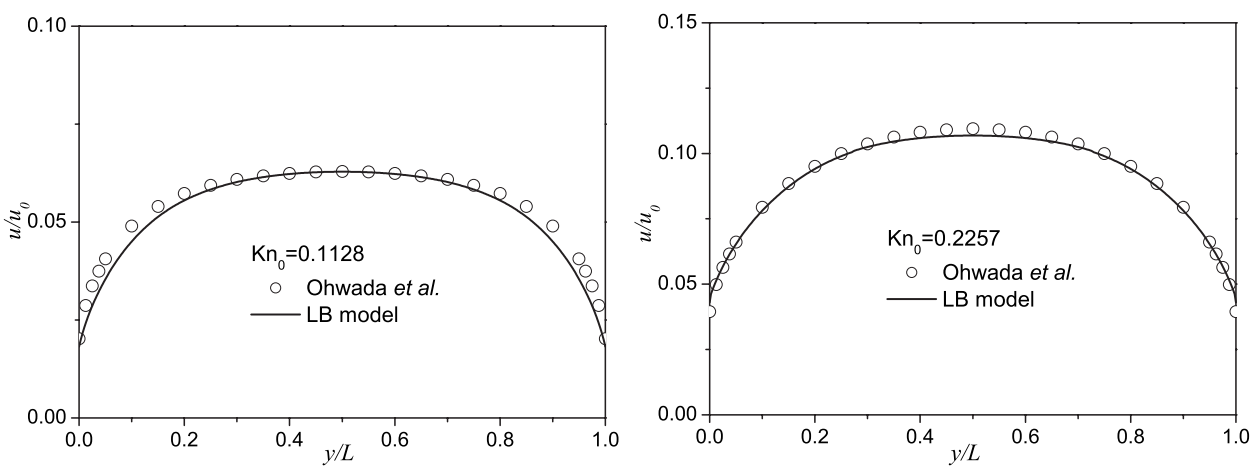

(a)

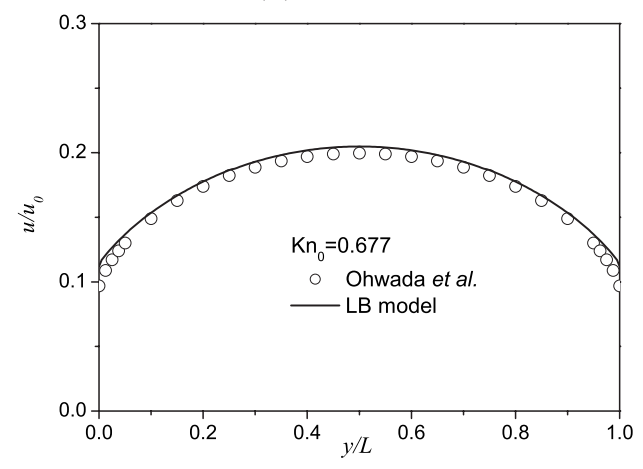

(c) (b)

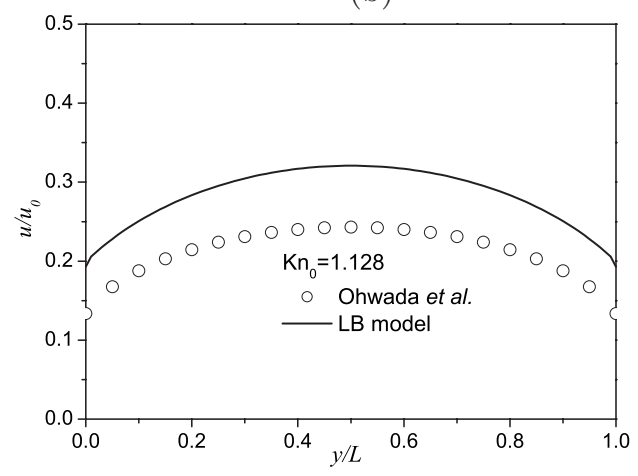

(d)
FIG. 2. Velocity profiles for thermal creep flow between two parallel plates at (a) $\mathrm{Kn}_{0}=0.1128$, (b) $\mathrm{Kn}_{0}=0.2257$, (c) $\mathrm{Kn}_{0}=0.677$, and (d) $\mathrm{Kn}_{0}=1.128$. The symbols represent the solution of the linearized Boltzmann equation presented by Owhada et al. [20], and the velocities have been normalized by the reference velocity $u_{0}$ $=\beta \sqrt{2 R T_{0}} . \quad\left[T_{0}\right.$ is the reference temperature and $\beta$ is a nondimensional temperature gradient-i.e., $\partial\left(T / T_{0}\right) / \partial(x / L)$, where $x$ is the distance in the flow direction. The chosen temperature gradient must be small to ensure consistency with the solution of the linearized Boltzmann equation.] 
indicating that the proposed model can capture thermal transpiration phenomena in the slip flow regime and part of the transition regime. However, discrepancies between our predictions and the linearized Boltzmann solution become significant when the Knudsen number is greater than unity. The present work suggests that our proposed lattice Boltzmann model is valid for Knudsen numbers up to $\mathrm{Kn} \sim O(1)$. To the best of the authors' knowledge, this is the first successful attempt at modeling thermal transpiration using a lattice Boltzmann approach. In addition, the computational efficiency of the proposed method is comparable to a NavierStokes solver, making it a practical simulation tool for nonequilibrium thermal flows in industrially-relevant geometries.

\section{CONCLUSIONS}

A thermal lattice Boltzmann model incorporating two distribution functions has been proposed for modeling thermally induced flows in the slip and transition regimes. The model has been shown to give good agreement with data obtained by the direct simulation Monte Carlo method and direct solution of the Boltzmann equation for Knudsen numbers up to $\mathrm{Kn} \sim O(1)$. The main advantages of the present LB method include the small number of discrete velocities, its simple algorithm, and its numerical efficiency compared to kinetic approaches. In conclusion, the thermal lattice Boltzmann model provides an efficient and cost-effective modeling tool for nonequilibrium gas flows in micro- and nanofluidic devices.

\section{ACKNOWLEDGMENTS}

The authors would like to thank Dr. Michael Gallis of Sandia National Laboratories for kindly providing the DSMC data. We would also like to thank Professor Jason Reese from the University of Strathclyde for many informative discussions. This work was financially supported by the Engineering and Physical Sciences Research Council (U.K.) (EPSRC) under Grants No. EP/D07455X/1 and No. EP/ F028865/1. Additional support was provided by EPSRC under the auspices of Collaborative Computational Project 12.
[1] O. Reynolds, Philos. Trans. R. Soc. London 170, 727 (1879).

[2] J. C. Maxwell, Philos. Trans. R. Soc. London 170, 231 (1879).

[3] E. H. Kennard, Kinetic theory of gases (McGraw-Hill, New York, 1938).

[4] E. P. Muntz, Y. Sone, K. Aoki, S. Vargo, and M. Young, J. Vac. Sci. Technol. A 20, 214 (2002).

[5] Y. L. Han, E. P. Muntz, A. Alexeenko, and M. Young, Nanoscale Microscale Thermophys. Eng. 11, 151 (2007).

[6] Y. Sone, Annu. Rev. Fluid Mech. 32, 779 (2000).

[7] R. Benzi, S. Succi, and M. Vergassola, Phys. Rep. 222, 145 (1992).

[8] S. Chen and G. D. Doolen, Annu. Rev. Fluid Mech. 30, 329 (1998).

[9] X. W. Shan, X. F. Yuan, and H. D. Chen, J. Fluid Mech. 550, 413 (2006).

[10] X. Y. He, S. Chen, and G. D. Doolen, J. Comput. Phys. 146, 282 (1998).
[11] G. H. Tang, Y. H. Zhang, and D. R. Emerson, Phys. Rev. E 77, 046701 (2008).

[12] Y. H. Zhang, X. J. Gu, R. W. Barber, and D. R. Emerson, EPL 77, 30003 (2007).

[13] Y. Shi, T. S. Zhao, and Z. L. Guo, Phys. Rev. E 70, 066310 (2004).

[14] S. S. Chikatamarla and I. V. Karlin, Phys. Rev. Lett. 97, 190601 (2006).

[15] G. H. Tang, Y. H. Zhang, X. J. Gu, and D. R. Emerson, EPL 83, 40008 (2008).

[16] D. W. Stops, J. Phys. D 3, 685 (1970).

[17] Z. L. Guo, B. C. Shi, and C. G. Zheng, EPL 80, 24001 (2007).

[18] S. Ansumali and I. V. Karlin, Phys. Rev. E 66, 026311 (2002).

[19] M. A. Gallis, D. J. Rader, and J. R. Torczynski, Phys. Fluids 14, 4290 (2002).

[20] T. Ohwada, Y. Sone, and K. Aoki, Phys. Fluids A 1, 2042 (1989). 